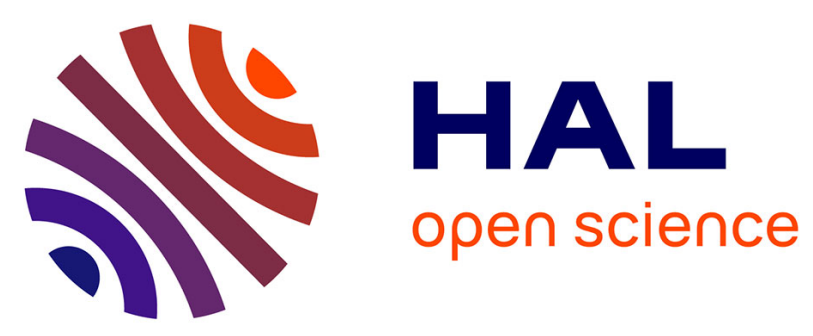

\title{
Robust Relay Beamforming for MIMO Multi-Relay Networks with Imperfect Channel Estimation
}

\author{
Zijian Wang, Wen Chen, Benoit Geller, Olivier Rioul
}

\section{To cite this version:}

Zijian Wang, Wen Chen, Benoit Geller, Olivier Rioul. Robust Relay Beamforming for MIMO MultiRelay Networks with Imperfect Channel Estimation. Global Communications Conference (GLOBECOM), 2013, Atlanta, United States. 10.1109/GLOCOM.2013.6831574 . hal-01227422

HAL Id: hal-01227422

https://hal-ensta-paris.archives-ouvertes.fr/hal-01227422

Submitted on 4 Dec 2015

HAL is a multi-disciplinary open access archive for the deposit and dissemination of scientific research documents, whether they are published or not. The documents may come from teaching and research institutions in France or abroad, or from public or private research centers.
L'archive ouverte pluridisciplinaire HAL, est destinée au dépôt et à la diffusion de documents scientifiques de niveau recherche, publiés ou non, émanant des établissements d'enseignement et de recherche français ou étrangers, des laboratoires publics ou privés. 


\section{Robust Relay Beamforming for MIMO Multi-Relay Networks with Imperfect Channel Estimation}

\author{
Zijian Wang, and, Wen Chen, \\ Department of Electronic Engineering \\ Shanghai Jiao Tong University \\ Shanghai, China 200240
}

\author{
Benoit Geller \\ U2IS ENSTA ParisTech \\ 828 boulevard des marechaux \\ 91762 Palaiseau Cedex, France
}

\author{
Olivier Rioul \\ LTCI Comelec Telecoms ParisTech \\ 46, rue Barrault \\ 75634 Paris Cedex 13, France
}

\begin{abstract}
In this paper, we consider a dual-hop Multiple Input Multiple Output (MIMO) wireless multi-relay network, in which a source-destination pair both equipped with multiple antennas communicates through multiple half-duplex amplify-and-forward (AF) relay terminals which are also with multiple antennas. Imperfect channel estimations for all nodes are considered. We propose a novel robust linear beamforming at the relays, based on the QR decomposition filter at the destination node which performs successive interference cancellation (SIC). Using Law of Large Number, we obtain the asymptotic rate, upon which the proposed relay beamforming is optimized. Simulation results show that the asymptotic rate matches with the ergodic rate well. Analysis and simulation results demonstrate that the proposed beamforming outperforms the conventional beamforming schemes.
\end{abstract}

Index Terms-MIMO relay, successive interference cancellation (SIC) detection, relay beamforming, channel estimation, rate.

\section{INTRODUCTION}

MIMO relay network has been of great interest in recent years as it allows an increase of the data rate in the cellular edge and extend the network coverage [1], [2].

In [3], the authors show a scaling theory for multi-relay MIMO network. In [4], [5], the authors design three relay beamforming schemes based on matrix triangularization which have superiority over the conventional zero-forcing (ZF) and amplify-and-forward (AF) beamformers. The proposed beamforming scheme can both fulfill intranode gain and distributed array gain. [6] designed a beamforming scheme that achieves the upper bound of capacity with a small gap when $K$ is significantly large. But it has bad performance for small $K$ and the source needs channel state information(CSI) which increases overhead. In [7], two efficient relay-beamformers for the dual-hop MIMO multi-relay networks have been presented, which are based on matched filter (MF) and regularized zeroforcing (RZF). But the beamformers are not optimized to the network. In [8], the authors investigated lower and upper bounds of mutual information under CSI error. In [9], the authors studied the trade-off between accuracy of channel estimation and data transmission, and show that the optimal number of training symbols is equal to the number of transmit antennas. Imperfect CSI for MIMO relay networks were taken into account in [10]-[12].
In this paper, we propose a new robust beamforming scheme for dual-hop MIMO multi-relay networks with imperfect channel estimation. SIC is also implemented at the destination by $\mathrm{QR}$ decomposition. The proposed beamformer at relay is based on the MMSE receiver and the RZF precoder. We optimize their regularizing factors. In the derivation, using the Law of Large Number, we obtain the asymtotic rate capacity for the MMSE-RZF beamformer, based on which, the performance of the beamformer can be easily analyzed. Simulation results show that the asymptotic rate capacity matches well the ergodic capacity. The asymptotic rate also validates the scaling law in [3]. Analysis and simulations demonstrate that the rate of our robust MMSE-RZF outperforms other published schemes.

In this paper, boldface lowercase letter and boldface uppercase letter represent vectors and matrices, respectively. Notations $(\mathbf{A})_{i}$ and $(\mathbf{A})_{i, j}$ denote the $i$-th row and $(i, j)$-th entry of the matrix A. Notations $\operatorname{tr}(\cdot),(\cdot)^{\dagger},(\cdot)^{*}$ and $(\cdot)^{H}$ denote trace, pseudo-inverse, conjugate and conjugate transpose operation of a matrix respectively. Term $\mathbf{I}_{N}$ is an $N \times N$ identity matrix. The $\operatorname{diag}\left\{\left\{a_{m}\right\}_{m=1}^{M}\right\}$ denotes a diagonal matrix with diagonal entries of $a_{1}, \ldots, a_{M} .\|\mathbf{a}\|$ stands for the Euclidean norm of a vector $\mathbf{a}$, and $\stackrel{w \cdot p_{\dot{p}}}{\longrightarrow}$ represents convergence with probability one. Finally, we denote the expectation operation by $\mathrm{E}\{\cdot\}$.

\section{SySTEM MODEL}

The considered MIMO multi-relay network consists of a single source and destination node both equipped with $M$ antennas, and $K N$-antenna relay nodes distributed between the source-destination pair. We consider half-duplex nonregenerative relaying throughout this paper, where it takes two non-overlapping time slots for the data to be transmitted from the source to the destination node via the backward channels and forward channels. Due to deep large-scale fading effects produced by the long distance, we assume that there is no direct link between the source and destination. Imperfect estimations are assumed. Assuming that $\widehat{\mathbf{H}}_{k} \in \mathbb{C}^{M \times M}$ and $\widehat{\mathbf{G}}_{k} \in \mathbb{C}^{M \times M}$ stands for the available imperfect CSIs of backward channel (BC) and forward channel (FC), we model 
the channel as

$$
\begin{aligned}
& \mathbf{H}_{k}=\widehat{\mathbf{H}}_{k}+e_{1} \boldsymbol{\Omega}_{1, k}, \\
& \mathbf{G}_{k}=\widehat{\mathbf{G}}_{k}+e_{2} \boldsymbol{\Omega}_{2, k},
\end{aligned}
$$

where $\mathbf{H}_{k} \in \mathbb{C}^{M \times M}$ and $\mathbf{G}_{k} \in \mathbb{C}^{M \times M}(k=1, \ldots, K)$ stand for the real CSI. $\boldsymbol{\Omega}_{1, k}$ and $\boldsymbol{\Omega}_{2, k}$ are matrices respectively independent of $\mathbf{H}_{k}$ and $\mathbf{G}_{k}$, whose entries are independently and identically distributed(i.i.d) zero-mean complex Gaussian, with unity variance [8], [13]. Therefore the power of CSI errors of $\mathrm{BC}$ and $\mathrm{FC}$ are $e_{1}^{2}$ and $e_{2}^{2}$. All channels $\mathbf{H}_{1}, \cdots, \mathbf{H}_{K}$ and $\mathbf{G}_{1}, \cdots, \mathbf{G}_{K}$ can be supposed to be i.i.d and experience the same Rayleigh flat fading.

In the first time slot, the source node broadcasts the signal to all the relay nodes through BCs. Let $M \times 1$ vector $\mathbf{s}$ be the transmit signal vector satisfying the power constraint $\mathrm{E}\left\{\mathbf{s s}^{H}\right\}=(P / M) \mathbf{I}_{M}$, where $P$ is defined as the transmit power at the source node. Then the corresponding received signal at the $k$-th relay can be written as $\mathbf{r}_{k}=\mathbf{H}_{k} \mathbf{s}+\mathbf{n}_{k}$, where the term $\mathbf{n}_{k}$ is the spatio-temporally white zero-mean complex additive Gaussian noise vector, independent across $k$, with the covariance matrix $\mathrm{E}\left\{\mathbf{n}_{k} \mathbf{n}_{k}^{H}\right\}=\sigma_{1}^{2} \mathbf{I}_{M}$. In the second time slot, firstly each relay node performs linear processing by multiplying $\mathbf{r}_{k}$ with an $N \times N$ beamforming matrix $\mathbf{F}_{k}$. This $\mathbf{F}_{k}$ is based on its imperfect CSIs $\widehat{\mathbf{H}}_{k}$ and $\widehat{\mathbf{G}}_{k}$. Consequently, the signal vector sent from the $k$-th relay node is $\mathbf{t}_{k}=\mathbf{F}_{k} \mathbf{r}_{k}$. From more practical consideration, we assume that each relay node has its own power constraint satisfying $\mathrm{E}\left\{\mathbf{t}_{k}^{H} \mathbf{t}_{k}\right\} \leq Q$, which is independent of power $P$. Hence a power constraint condition of $\mathbf{t}_{k}$ can be derived as

$$
\rho\left(\mathbf{t}_{k}\right)=\operatorname{tr}\left\{\mathbf{F}_{k}\left(\frac{P}{M} \mathbf{H}_{k} \mathbf{H}_{k}^{H}+\sigma_{1}^{2} \mathbf{I}_{N}\right) \mathbf{F}_{k}^{H}\right\} \leq Q .
$$

After linear relay beamforming processing, all the relay nodes forward their data simultaneously to the destination. Thus the signal vector received by the destination can be expressed as

$$
\mathbf{y}=\sum_{k=1}^{K} \mathbf{G}_{k} \mathbf{t}_{k}+\mathbf{n}_{d}=\sum_{k=1}^{K} \mathbf{G}_{k} \mathbf{F}_{k} \mathbf{H}_{k} \mathbf{s}+\sum_{k=1}^{K} \mathbf{G}_{k} \mathbf{F}_{k} \mathbf{n}_{k}+\mathbf{n}_{d},
$$

where $\mathbf{n}_{d} \in \mathbb{C}^{M}$, satisfying $\mathrm{E}\left\{\mathbf{n}_{d} \mathbf{n}_{d}^{H}\right\}=\sigma_{2}^{2} \mathbf{I}_{M}$, denotes the zero-mean white circularly symmetric complex additive Gaussian noise vector at the destination node with the noise power $\sigma_{2}^{2}$.

\section{RELAY BEAMForming DESIGN}

In this section, the QR detector at the destination node for SIC detection is introduced and a relay beamforming scheme based on MMSE receiver and RZF precoder is proposed.

\section{A. QR Decomposition and SIC Detection}

QR-decomposition (QRD) detector is utilized as the destination receiver $\mathbf{W}$ in this paper, which is proved to be asymptotically equivalent to that of the maximum-likelihood detector (MLD) [14]. Let $\sum_{k=1}^{K} \widehat{\mathbf{G}}_{k} \mathbf{F}_{k} \widehat{\mathbf{H}}_{k}=\mathbf{H}_{\mathcal{S D}}$. Then (4) can be rewritten as $\mathbf{y}=\mathbf{H}_{\mathcal{S D}} \mathbf{S}+\widehat{\mathbf{n}}$, where $\mathbf{H}_{\mathcal{S D}}$ represents the effective channel between the source and destination node, and

$\widehat{\mathbf{n}} \cong \sum_{k=1}^{K} e_{1} \widehat{\mathbf{G}}_{k} \mathbf{F}_{k} \boldsymbol{\Omega}_{1, k} \mathbf{s}+\sum_{k=1}^{K} e_{2} \boldsymbol{\Omega}_{2, k} \mathbf{F}_{k} \widehat{\mathbf{H}}_{k} \mathbf{s}+\sum_{k=1}^{K} \mathbf{G}_{k} \mathbf{F}_{k} \mathbf{n}_{k}+\mathbf{n}_{d}$

is the effective noise vector cumulated from the CSI errors, the noise $\mathbf{n}_{\mathbf{k}}$ at the $k$-th relay node, and the noise vector $\mathbf{n}_{d}$ at the destination. In the derivation, we omit the small term including $e_{1} e_{2}$. Finally, in order to cancel the interference from other antennas, QR decomposition of the effective channel is implemented as

$$
\mathbf{H}_{\mathcal{S D}}=\mathbf{Q}_{\mathcal{S D}} \mathbf{R}_{\mathcal{S D}}
$$

where $\mathbf{Q}_{\mathcal{S D}}$ is an $M \times M$ unitary matrix and $\mathbf{R}_{\mathcal{S D}}$ is an $M \times M$ right upper triangular matrix. Therefore the $\mathrm{QRD}$ detector at destination node is chosen as: $\mathbf{W}=\mathbf{Q}_{\mathcal{S} \mathcal{D}}^{H}$, and the signal vector after QRD detection becomes

$$
\tilde{\mathbf{y}}=\mathbf{Q}_{\mathcal{S D}}^{H} \mathbf{y}=\mathbf{R}_{\mathcal{S D}} \mathbf{s}+\mathbf{Q}_{\mathcal{S} \mathcal{D}}^{H} \widehat{\mathbf{n}} .
$$

A power control factor $\rho_{k}$ is set with $\mathbf{F}_{\mathbf{k}}$ in (3) to guarantee that the $k$-th relay transmit power is equal to $Q$. The transmit signal from each relay node after linear beamforming and power control becomes $\mathbf{t}_{k}=\rho_{k} \mathbf{F}_{k} \mathbf{r}_{k}$, where the power control factor $\rho_{k}$ can be derived from (3) as

$$
\rho_{k}=\left(Q / \mathrm{E}\left[\operatorname{tr}\left\{\mathbf{F}_{k}\left(\frac{P}{M} \mathbf{H}_{k} \mathbf{H}_{k}^{H}+\sigma_{1}^{2} \mathbf{I}_{N}\right) \mathbf{F}_{k}^{H}\right\}\right]\right)^{\frac{1}{2}} .
$$

\section{B. Beamforming at Relay Nodes}

We propose at the relay nodes a robust MMSE-RZF beamformer which can be optimized according to the network condition. We design the Beamforming at the $k$-th relay as follows

$\mathbf{F}_{k}=\widehat{\mathbf{G}}_{k}^{H}\left(\widehat{\mathbf{G}}_{k} \widehat{\mathbf{G}}_{k}^{H}+\alpha_{k}^{\mathrm{RZF}} \mathbf{I}_{M}\right)^{-1}\left(\widehat{\mathbf{H}}_{k}^{H} \widehat{\mathbf{H}}_{k}+\alpha_{k}^{\mathrm{MMSE}} \mathbf{I}_{M}\right)^{-1} \widehat{\mathbf{H}}_{k}^{H}$.

Note that MF-MF and ZF-ZF are two extreme cases for $\alpha_{k}^{\mathrm{MMSE}}=\alpha_{k}^{\mathrm{RZF}}=\infty$ and $\alpha_{k}^{\mathrm{MMSE}}=\alpha_{k}^{\mathrm{RZF}}=0$ respectively. Generally, if the $\alpha$ (either regularizing factor in MMSE or RZF) is too large, the effective channel matrix will far deviate from a diagonal matrix, which results in power consumption and interference across different datas. If $\alpha$ is too small, the MMSE receiver and RZF precoder will perform like a ZF receiver or precoder which have the power penalty problem due to its inverse Wishart distribution term in its transmit power [15]. We aim to obtain the optimal $\alpha_{k}^{\mathrm{MMSE}}$ and $\alpha_{k}^{\mathrm{RZF}}$ to maximize the rate in this paper. However, to directly get the global optimal closed-form solution is difficult. In the following, we derive an optimized solution by two steps. We first derive an optimized $\alpha_{k}^{\mathrm{MMSE}}$ by maximizing the SINR at the relay nodes, and then we derive an optimized $\alpha_{k}^{\mathrm{RZF}}$ dependent on the given optimized $\alpha_{k}^{\mathrm{MMSE}}$ by maximizing the rate at the destination. 


\section{Robust MMSE-RZF BEAMFORMER}

In this section, we derive the optimized $\alpha_{k}^{\mathrm{MMSE}}$ and $\alpha_{k}^{\mathrm{RZF}}$ in the MMSE-RZF beamformer by two steps. We first derive the optimized $\alpha_{k}^{\mathrm{MMSE}}$ by maximizing the SINR at relay nodes, and then derive the optimized $\alpha_{k}^{\mathrm{RZF}}$ for a given $\alpha_{k}^{\mathrm{MMSE}}$ based on the asymptotic rate.

\section{A. Optimization of $\alpha_{k}^{\mathrm{MMSE}}$}

We optimize $\alpha_{k}^{\text {MMSE }}$ by maximizing the SINR at relay nodes. For the $k$-th relay, the signal vector after processed by an MMSE receiver is

$$
\begin{aligned}
\mathbf{v}_{k} & =\left(\widehat{\mathbf{H}}_{k}^{H} \widehat{\mathbf{H}}_{k}+\alpha_{k}^{\mathrm{MMSE}} \mathbf{I}_{M}\right)^{-1} \widehat{\mathbf{H}}_{k}^{H} \mathbf{r}_{k} \\
& =\left(\widehat{\mathbf{H}}_{k}^{H} \widehat{\mathbf{H}}_{k}+\alpha_{k}^{\mathrm{MMSE}} \mathbf{I}_{M}\right)^{-1} \widehat{\mathbf{H}}_{k}^{H} \widehat{\mathbf{H}}_{k} \mathbf{S} \\
& +e_{1}\left(\widehat{\mathbf{H}}_{k}^{H} \widehat{\mathbf{H}}_{k}+\alpha_{k}^{\mathrm{MMSE}} \mathbf{I}_{M}\right)^{-1} \widehat{\mathbf{H}}_{k}^{H} \mathbf{\Omega}_{1, k} \mathbf{S} \\
& +\left(\widehat{\mathbf{H}}_{k}^{H} \widehat{\mathbf{H}}_{k}+\alpha_{k}^{\mathrm{MMSE}} \mathbf{I}_{M}\right)^{-1} \widehat{\mathbf{H}}_{k}^{H} \mathbf{n}_{k} .
\end{aligned}
$$

The first term in (10) is the signal vector, which contains inter-stream interference, because matrix $\left(\widehat{\mathbf{H}}_{k}^{H} \widehat{\mathbf{H}}_{k}+\alpha_{k}^{\mathrm{MMSE}} \mathbf{I}_{M}\right)^{-1} \widehat{\mathbf{H}}_{k}^{H} \widehat{\mathbf{H}}_{k}$ is not diagonal if $\alpha_{k}^{\mathrm{MMSE}} \neq 0$. So we need to calculate the power of desired signal and the interference. We use the diagonal decompositions in the following analysis, i.e.,

$$
\begin{aligned}
\widehat{\mathbf{H}}_{k} \widehat{\mathbf{H}}_{k}^{H} & =\mathbf{P}_{k} \operatorname{diag}\left\{\theta_{k, 1}, \ldots, \theta_{k, M}\right\} \mathbf{P}_{k}{ }^{H}, \\
\widehat{\mathbf{G}}_{k} \widehat{\mathbf{G}}_{k}^{H} & =\mathbf{Q}_{k} \operatorname{diag}\left\{\lambda_{k, 1}, \ldots, \lambda_{k, M}\right\} \mathbf{Q}_{k}{ }^{H},
\end{aligned}
$$

where $\mathbf{P}_{k}$ and $\mathbf{Q}_{k}$ are unitary matrices. Here we define that $\operatorname{diag}\left\{\theta_{k, 1}, \ldots, \theta_{k, M}\right\} \triangleq \boldsymbol{\Theta}_{k}$ and $\operatorname{diag}\left\{\lambda_{k, 1}, \ldots, \lambda_{k, M}\right\} \triangleq \boldsymbol{\Lambda}_{k}$. To divides the interference from the desired signal, we introduce the following two lemmas.

Lemma 1: Assume $\mathbf{A} \in \mathbb{C}^{M \times M}$ is a random matrix. If there is a diagonal decomposition $\mathbf{A}=\mathbf{Q} \mathbf{\Lambda} \mathbf{Q}^{H}$, where $\mathbf{\Lambda}=$ $\operatorname{diag}\left\{\lambda_{1}, \ldots, \lambda_{M}\right\} \in \mathbb{R}^{M \times M}$ and the matrix $\mathbf{Q}$ is unitary, we have

$\mathrm{E}\left\{(\mathbf{A})_{m, m}^{2}\right\}=\frac{1}{M(M+1)}\left(\left(\sum_{l=1}^{M} \lambda_{l}\right)^{2}+\sum_{l=1}^{M} \lambda_{l}^{2}\right) \triangleq \mu(\lambda)$,

for any $m$, where the expectation is taken over the distribution Q.

The proof of Lemma 1 can be directly obtained from [15] which considers perfect CSI. Due to the limited space, we omit the details.

Lemma 2: Assume $\mathbf{A} \in \mathbb{C}^{M \times M}$ is a random matrix. If there is a diagonal decomposition $\mathbf{A}=\mathbf{Q} \mathbf{\Lambda} \mathbf{Q}^{H}$, with $\boldsymbol{\Lambda}=$ $\operatorname{diag}\left\{\lambda_{1}, \ldots, \lambda_{M}\right\} \in \mathbb{R}^{M \times M}$ and unitary matrix $\mathbf{Q}$, we have

$$
\begin{aligned}
& \mathrm{E}\left\{\left|(\mathbf{A})_{m, j}\right|^{2}\right\}=\frac{1}{(M-1)(M+1)} \sum_{l=1}^{M} \lambda_{l}^{2} \\
& -\frac{1}{(M-1) M(M+1)}\left(\sum_{l=1}^{M} \lambda_{l}\right)^{2} \triangleq \nu(\lambda),
\end{aligned}
$$

for any $m \neq j$, where the expectation is taken over the distribution $\mathbf{Q}$.

Proof: Because A is a conjugate symmetric matrix, we have

$$
\begin{aligned}
& \mathrm{E}\left\{\sum_{j=1, j \neq m}^{M}\left|(\mathbf{A})_{m, j}\right|^{2}\right\}+\mathrm{E}\left\{(\mathbf{A})_{m, m}^{2}\right\} \\
= & \mathrm{E}\left\{\left(\mathbf{A} \mathbf{A}^{H}\right)_{m, m}\right\}=\mathrm{E}\left\{\left(\mathbf{Q} \mathbf{\Lambda}^{2} \mathbf{Q}^{H}\right)_{m, m}\right\}=\frac{1}{M} \sum_{l=1}^{M} \lambda_{l}^{2} .
\end{aligned}
$$

Since $\mathrm{E}\left\{\left|(\mathbf{A})_{k, j}\right|^{2}\right\}$ are all equal for $j \neq k$, we have

$$
\begin{aligned}
& \mathrm{E}\left\{\left|(\mathbf{A})_{k, j}\right|^{2}\right\}=\frac{1}{(M-1)}\left(\frac{1}{M} \sum_{l=1}^{M} \lambda_{l}^{2}-\mathrm{E}\left\{(\mathbf{A})_{m, m}^{2}\right\}\right) \\
= & \frac{1}{(M-1)(M+1)} \sum_{l=1}^{M} \lambda_{l}^{2}-\frac{1}{(M-1) M(M+1)}\left(\sum_{l=1}^{M} \lambda_{l}\right)^{2} .
\end{aligned}
$$

Now we return to derive the signal-to-interference noise ratio (SINR) for each stream at each relay. The first term in the right hand side of (10) can be rewritten as

$$
\left(\widehat{\mathbf{H}}_{k}^{H} \widehat{\mathbf{H}}_{k}+\alpha_{k}^{\mathrm{MMSE}} \mathbf{I}_{M}\right)^{-1} \widehat{\mathbf{H}}_{k}^{H} \widehat{\mathbf{H}}_{k} \mathbf{s}=\mathbf{P}_{k} \frac{\boldsymbol{\Theta}_{k}}{\boldsymbol{\Theta}_{k}+\alpha_{k}^{\mathrm{MMSE}} \mathbf{I}_{N}} \mathbf{P}_{k}{ }^{H} \mathbf{s} .
$$

Therefore, from Lemma 1, the power of the desired signal of the $m$-th stream can be calculated as

$$
\begin{aligned}
\mathrm{E}\left\{\left|\left(\mathbf{P}_{k} \frac{\boldsymbol{\Theta}_{k}}{\boldsymbol{\Theta}_{k}+\alpha_{k}^{\mathrm{MMSE}} \mathbf{I}_{N}} \mathbf{P}_{k}{ }^{H}\right)_{m, m} \mathbf{s}_{m}\right|^{2}\right\} \\
=\frac{P}{M} \mu\left(\frac{\theta_{k}}{\theta_{k}+\alpha_{k}^{\mathrm{MMSE}}}\right),
\end{aligned}
$$

where $\theta_{k}$ denotes the set of all the diagonal entries in $\boldsymbol{\Theta}_{k}$. From Lemma 2, the interference from other streams are

$$
\begin{array}{r}
\mathrm{E}\left\{\left|\sum_{j=1, j \neq m}^{M}\left(\mathbf{P}_{k} \frac{\boldsymbol{\Theta}_{k}}{\boldsymbol{\Theta}_{k}+\alpha_{k}^{\mathrm{MMSE}} \mathbf{I}_{N}} \mathbf{P}_{k}{ }^{H}\right)_{m, j} \mathbf{s}_{j}\right|^{2}\right\} \\
=\frac{P(M-1)}{M} \nu\left(\frac{\theta_{k}}{\theta_{k}+\alpha_{k}^{\mathrm{MMSE}}}\right) .
\end{array}
$$

The effective noise of the $m$-th stream is

$$
\begin{aligned}
\mathbf{n}_{\mathrm{eff}, k}=e_{1}\left(\widehat{\mathbf{H}}_{k}^{H} \widehat{\mathbf{H}}_{k}+\alpha_{k}^{\mathrm{MMSE}} \mathbf{I}_{M}\right)^{-1} \widehat{\mathbf{H}}_{k}^{H} \boldsymbol{\Omega}_{1, k} \mathbf{s} \\
+\left(\widehat{\mathbf{H}}_{k}^{H} \widehat{\mathbf{H}}_{k}+\alpha_{k}^{\mathrm{MMSE}} \mathbf{I}_{M}\right)^{-1} \widehat{\mathbf{H}}_{k}^{H} \mathbf{n}_{k},
\end{aligned}
$$

whose covariance matrix can be similarly calculated as

$$
\mathrm{E}\left\{\mathbf{n}_{\mathrm{eff}, k} \mathbf{n}_{\mathrm{eff}, k}^{H}\right\}=\frac{e_{1}^{2} P+\sigma_{1}^{2}}{M} \sum_{m=1}^{M} \frac{\theta_{k, m}}{\left(\theta_{k, m}+\alpha_{k}^{\mathrm{MMSE}}\right)^{2}} \mathbf{I}_{M},
$$


where we used the fact $\mathrm{E}\left\{\boldsymbol{\Omega} \mathbf{A} \boldsymbol{\Omega}^{H}\right\}=\operatorname{tr}(\mathbf{A})$ for an $N \times N$ matrix A. In (18), (19) and (21), expectations are taken over their respective unitary matrices. Combining (18), (19), and (21), the SINR of the $m$-th stream at the $k$-th relay is

$$
\frac{\frac{P}{M} \mu\left(\frac{\theta_{k}}{\theta_{k}+\alpha_{k}^{\mathrm{MMSE}}}\right)}{\frac{P(M-1)}{M} \nu\left(\frac{\theta_{k}}{\theta_{k}+\alpha_{k}^{\mathrm{MMSE}}}\right)+\frac{e_{1}^{2} P+\sigma_{1}^{2}}{M} \sum_{m=1}^{M} \frac{\theta_{k, m}}{\left(\theta_{k, m}+\alpha_{k}^{\mathrm{MMSE}}\right)^{2}}} .
$$

The derived SINR in (22) is a function of the singular values of the instantaneous channel. To maximize the SINR expression, we introduce the following lemma which is a conclusion of the Appendix B in [15].

Lemma 3: For an SNR in terms of $\alpha$,

$$
=\frac{\operatorname{SNR}(\alpha)}{C \sum_{l=1}^{M} \frac{\lambda_{l}}{\left(\lambda_{l}+\alpha\right)^{2}}+D \sum_{l=1}^{M} \frac{\lambda_{l}^{2}}{\left(\lambda_{l}+\alpha\right)^{2}}+E\left(\sum_{l=1}^{M} \frac{\lambda_{l}}{\lambda_{l}+\alpha}\right)^{2}},
$$

is maximized by $\alpha=C / D$.

The optimum value of $\alpha$ can be obtained by differentiating (23) and setting it to be zero, which results in

$$
\sum_{l>k} \frac{\lambda_{l} \lambda_{k}\left(\lambda_{k}-\lambda_{l}\right)^{2}(C / D-\alpha)}{\left(\lambda_{l}+\alpha\right)^{3}\left(\lambda_{k}+\alpha\right)^{3}}=0 .
$$

For the eigenvalues are not all equal, the SINR is maximized only when $\alpha=C / D$. Substituting $\mu(\lambda)$ and $\nu(\lambda)$ into (22) and using Lemma 3, we obtain

$\alpha^{\mathrm{MMSE}, \mathrm{opt}}=\frac{\frac{e_{1}^{2} P+\sigma_{1}^{2}}{M}}{\frac{1}{(M-1)(M+1)} \cdot \frac{P(M-1)}{M}}=(M+1)\left(e_{1}^{2}+\frac{\sigma_{1}^{2}}{P}\right)$.

We see that the derived $\alpha_{k}^{\mathrm{MMSE}, \mathrm{opt}}$ is a closed-form value independent of the instantaneous channel.

\section{B. Optimization of $\alpha_{k}^{\mathrm{RZF}}$}

To optimize $\alpha_{k}^{\mathrm{RZF}}$, we need to derive the rate of the system. In the rest of the analysis, we write $\mathbf{F}_{k}^{\mathrm{MMSE}-\mathrm{RZF}}$ in (9) as $\mathbf{F}_{k}$ for simplicity. By adding the power control factor at the relays, we have

$$
\mathbf{H}_{\mathcal{S D}}=\sum_{k=1}^{K} \rho_{k} \widehat{\mathbf{G}}_{k} \mathbf{F}_{k} \widehat{\mathbf{H}}_{k} .
$$

The effective noise vector in (5) is

$$
\begin{aligned}
\widehat{\mathbf{n}}=\sum_{k=1}^{K} e_{1} \rho_{k} \widehat{\mathbf{G}}_{k} \mathbf{F}_{k} \boldsymbol{\Omega}_{1, k} \mathbf{s} & +\sum_{k=1}^{K} e_{2} \rho_{k} \boldsymbol{\Omega}_{2, k} \mathbf{F}_{k} \widehat{\mathbf{H}}_{k} \mathbf{s} \\
& +\sum_{k=1}^{K} \rho_{k} \mathbf{G}_{k} \mathbf{F}_{k} \mathbf{n}_{k}+\mathbf{n}_{d} .
\end{aligned}
$$

Finally we obtain the SNR of the $m$-th data stream at the destination after $\mathrm{QR}$ decomposition as

$$
\mathrm{SNR}_{m}^{\mathrm{D}}=\frac{\frac{P}{M}\left|\left(\mathbf{R}_{\mathcal{S D}}\right)_{m, m}\right|^{2}}{\frac{P}{M} \sum_{j=m+1}^{M}\left|\left(\mathbf{R}_{\mathcal{S D}}\right)_{m, j}\right|^{2}+\left(\mathbf{N}_{\mathrm{cov}}\right)_{m, m}},
$$

where $\mathbf{N}_{\text {cov }}$ is the covariance matrix of $\widehat{\mathbf{n}}$ after $\mathrm{QR}$ decomposition of the effective channel. The ergodic rate is derived by summing up all the data rates on each antenna link, i.e.,

$$
C=\mathrm{E}_{\left\{\widehat{\mathbf{H}}_{k}, \widehat{\mathbf{G}}_{k}\right\}_{k=1}^{K}}\left\{\frac{1}{2} \sum_{m=1}^{M} \log _{2}\left(1+\mathrm{SNR}_{m}^{\mathrm{D}}\right)\right\},
$$

where the $\frac{1}{2}$ penalty is due to the two time-slot transmission. From (28), we see that it is difficult to obtain the optimal solution directly. We derive asymptotic rate for large $K$ and then get the optimized $\alpha_{k}^{\mathrm{RZF}}$. Since all terms in (26) and (27) include $\rho_{k}$ except for $\mathbf{n}_{\mathbf{d}}$, we first consider the expectation of $\rho_{k}^{-2}$. From (8), substituting the perfect CSIs with (1) and (2), and taking expectation over $\mathbf{P}_{k}$ and $\mathbf{Q}_{k}$ and leaving the expectations of $\lambda$ and $\theta$, we have

$$
\begin{gathered}
\rho^{-2} \triangleq \mathrm{E}\left\{\rho_{k}^{-2}\right\}=\frac{P}{Q} \mathrm{E}\left\{\frac{\theta^{2}}{\left(\theta+\alpha^{\mathrm{MMSE}}\right)^{2}}\right\} \mathrm{E}\left\{\frac{\lambda}{\left(\lambda+\alpha^{\mathrm{MMSE}}\right)^{2}}\right\} \\
+\frac{\left(e_{1}^{2} P+\sigma_{1}^{2}\right) M}{Q} \times \mathrm{E}\left\{\frac{\theta}{\left(\theta+\alpha^{\mathrm{MMSE}}\right)^{2}}\right\} \mathrm{E}\left\{\frac{\lambda}{\left(\lambda+\alpha^{\mathrm{MMSE}}\right)^{2}}\right\},
\end{gathered}
$$

where the expectation is taken over distributions of $\mathbf{P}_{k}$ and $\mathbf{Q}_{k}(k=1, \ldots, K)$. Here we denote $\alpha, \lambda$ and $\theta$ without subscript $k$ and $m$ for simplicity, because all the channels for different relays are i.i.d. and $\lambda_{m}$ and $\theta_{m}$ for every $m$ are identically distributed. (30) implies that the expectation of $\rho_{k}^{-2}$ results in a uniform fixed $\rho^{-2}$ for all relays. Therefore we approximate $\rho_{k}^{-2}$ by $\rho^{-2}$ in the following analysis. The performance with such approximation varies little compared with using the dynamic power control factors [16]. Since all terms in the numerator and the denominator of (28) excerpt for the $\mathbf{n}_{\mathbf{d}}$ will generate $\rho_{k}^{2}$, in the following analysis, we can omit $\rho_{k}$ in calculation and multiply $\rho^{-2}$ to $\sigma^{2}$ which is the power of $\mathbf{n}_{\mathbf{d}}$. For the case of large $K$, using Law of Large Number, we have the approximations

$$
\begin{aligned}
& \left(\mathbf{H}_{\mathcal{S D}}\right)_{i, i} \stackrel{w \cdot p_{i}}{\longrightarrow} K\left(\mathrm{E}\left\{\left(\widehat{\mathbf{G}}_{k} \mathbf{F}_{k} \widehat{\mathbf{H}}_{k}\right)_{i, i}\right\}\right) \\
= & K \mathrm{E}\left\{\left(\mathbf{Q}_{k} \frac{\boldsymbol{\Lambda}_{k}}{\boldsymbol{\Lambda}_{k}+\alpha^{\mathrm{RZF}} \mathbf{I}_{M}} \mathbf{Q}_{k}^{H} \mathbf{P}_{k} \frac{\boldsymbol{\Theta}_{k}}{\boldsymbol{\Theta}_{k}+\alpha^{\mathrm{MMSE}} \mathbf{I}_{M}} \mathbf{P}_{k}^{H}\right)_{i, i}\right\} \\
= & \frac{K}{M N} \mathrm{E}\left\{\sum_{m=1}^{M} \frac{\theta_{k, m}}{\theta_{k, m}+\alpha^{\mathrm{MMSE}}}\right\} \mathrm{E}\left\{\sum_{m=1}^{M} \frac{\lambda_{k, m}}{\lambda_{k, m}+\alpha^{\mathrm{RZF}}}\right\} \\
= & K \mathrm{E}\left\{\frac{\theta}{\theta+\alpha^{\mathrm{MMSE}}}\right\} \mathrm{E}\left\{\frac{\lambda}{\lambda+\alpha^{\mathrm{RZF}}}\right\},
\end{aligned}
$$

Note that

$$
\begin{aligned}
\mathrm{E}\{ & \left.\left(\widehat{\mathbf{G}}_{k} \mathbf{F}_{k} \widehat{\mathbf{H}}_{k}\right)_{i, j}\right\} \\
=\mathrm{E}\{ & \left.\left(\mathbf{Q}_{k} \frac{\boldsymbol{\Lambda}_{k}}{\boldsymbol{\Lambda}_{k}+\alpha^{\mathrm{RZF}} \mathbf{I}_{M}} \mathbf{Q}_{k}^{H} \mathbf{P}_{k} \frac{\boldsymbol{\Theta}_{k}}{\boldsymbol{\Theta}_{k}+\alpha^{\mathrm{MMSE}} \mathbf{I}_{M}} \mathbf{P}_{k}^{H}\right)_{i, j}\right\} \\
= & \sum_{l, m, n} \mathrm{E}\left\{\left(\mathbf{Q}_{k}\right)_{i, l}\left(\frac{\boldsymbol{\Lambda}_{k}}{\boldsymbol{\Lambda}_{k}+\alpha^{\mathrm{RZF}} \mathbf{I}_{M}}\right)_{l}\left(\mathbf{Q}_{k}^{H}\right)_{l, m}\right. \\
& \left.\left(\mathbf{P}_{k}\right)_{m, n}\left(\frac{\boldsymbol{\Theta}_{k}}{\boldsymbol{\Theta}_{k}+\alpha^{\mathrm{MMSE}} \mathbf{I}_{M}}\right)_{n}\left(\mathbf{P}_{k}^{H}\right)_{n, j}\right\}=0
\end{aligned}
$$


for $i \neq j$, because $\left(\mathbf{Q}_{k}\right)_{i, l}\left(\mathbf{Q}_{k}^{H}\right)_{l, m}=0$ for $i \neq m$ and $\left(\mathbf{P}_{k}\right)_{m, n}\left(\mathbf{P}_{k}^{H}\right)_{n, j}=0$ for $m \neq j$. Then we have

$$
\frac{\left(\mathbf{H}_{\mathcal{S D}}\right)_{i, j}}{K} \longrightarrow \mathrm{E}\left\{\left(\widehat{\mathbf{G}}_{k} \mathbf{F}_{k} \widehat{\mathbf{H}}_{k}\right)_{i, j}\right\}=0
$$

for large $K$. Therefore, from (31) and (33), we have $\left(\mathbf{H}_{\mathcal{S D}}\right)_{i, i}=O(K)$ and $\left(\mathbf{H}_{\mathcal{S D}}\right)_{i, j}=o(K)$, which results in that $\frac{\mathbf{H}_{\mathcal{S D}}}{K}$ is asymptotically diagonal for large $K$. So we have $\mathbf{Q}_{\mathcal{S D}} \stackrel{w \cdot p_{i}}{\longrightarrow} \mathbf{I}_{M}$ and $\mathbf{R}_{\mathcal{S D}} \stackrel{w \cdot p_{\dot{p}}}{\longrightarrow} \mathbf{H}_{\mathcal{S D}}$ for large $K$.

To obtain the power of interference, we calculate the nondiagonal entries of the effective channel matrix which is included in (37) in the appendix.

Since the fact in [15] that

$$
\begin{aligned}
& \mathrm{E}\left\{\left|\left(\mathbf{Q}_{r}\right)_{i, k}\right|^{2}\left|\left(\mathbf{Q}_{r}\right)_{l, k}\right|^{2}\right\}=\frac{2}{M(M+1)} \quad \text { if } i=l(34) \\
& \mathrm{E}\left\{\left|\left(\mathbf{Q}_{r}\right)_{i, k}\right|^{2}\left|\left(\mathbf{Q}_{r}\right)_{l, k}\right|^{2}\right\}=\frac{1}{M(M+1)} \quad \text { if } \quad i \neq l(35)
\end{aligned}
$$

then when $i \neq m, l \neq r$ we have

$$
\begin{gathered}
\mathrm{E}\left\{\left(\mathbf{Q}_{k}\right)_{i, l}\left(\mathbf{Q}_{k}\right)_{m, l}^{*}\left(\mathbf{Q}_{k}\right)_{i, r}^{*}\left(\mathbf{Q}_{k}\right)_{m, r}\right\} \\
=\frac{1}{M(M-1)} \mathrm{E}\left\{\sum_{l, r=1, l \neq r}^{M}\left(\mathbf{Q}_{k}\right)_{i, l}\left(\mathbf{Q}_{k}\right)_{m, l}^{*}\left(\mathbf{Q}_{k}\right)_{i, r}^{*}\left(\mathbf{Q}_{k}\right)_{m, r}\right\} \\
=\frac{1}{M(M-1)} \mathrm{E}\left\{\sum_{l, r=1}^{M}\left(\mathbf{Q}_{k}\right)_{i, l}\left(\mathbf{Q}_{k}\right)_{m, l}^{*}\left(\mathbf{Q}_{k}\right)_{i, r}^{*}\left(\mathbf{Q}_{k}\right)_{m, r}\right\} \\
-\frac{1}{M(M-1)} \mathrm{E}\left\{\sum_{l=1}^{M}\left|\left(\mathbf{Q}_{k}\right)_{i, l}\right|^{2}\left|\left(\mathbf{Q}_{k}\right)_{m, l}\right|^{2}\right\} \\
=-\frac{1}{(M-1) M(M+1)} .
\end{gathered}
$$

Using (34), (35) and (36), and through some manipulation, we have where $\lambda$ and $\lambda^{\prime}, \theta$ and $\theta^{\prime}$ are different singular values within one decomposition. Obviously, (37) equals zero when $\alpha^{\mathrm{MMSE}}$ and $\alpha^{\mathrm{RZF}}$ are zero. Let us define the following expectations. $\mathcal{E}_{1}^{\theta} \triangleq \mathrm{E}\left\{\frac{\theta}{\left(\theta+\alpha^{\mathrm{MMSE}}\right)}\right\}, \mathcal{E}_{2}^{\theta} \triangleq \mathrm{E}\left\{\frac{\theta}{\left(\theta+\alpha^{\mathrm{MMSE}}\right)^{2}}\right\}$, $\mathcal{E}_{3}^{\theta} \triangleq \mathrm{E}\left\{\frac{\theta^{2}}{\left(\theta+\alpha^{\mathrm{MMSE}}\right)^{2}}\right\}, \mathcal{E}_{4}^{\theta} \triangleq \mathrm{E}\left\{\frac{\theta \theta^{\prime}}{\left(\theta+\alpha^{\mathrm{MMSE}}\right)\left(\theta^{\prime}+\alpha^{\mathrm{MMSE}}\right)}\right\}$, and $\mathcal{E}_{1}^{\lambda} \triangleq \mathrm{E}\left\{\frac{\lambda}{\left(\lambda+\alpha^{\mathrm{RZF}}\right)}\right\}, \mathcal{E}_{2}^{\lambda} \triangleq \mathrm{E}\left\{\frac{\lambda}{\left(\lambda+\alpha^{\mathrm{RZF}}\right)^{2}}\right\}, \mathcal{E}_{3}^{\lambda \triangleq}$ $\mathrm{E}\left\{\frac{\lambda^{2}}{\left(\lambda+\alpha^{\mathrm{RZF}}\right)^{2}}\right\}, \mathcal{E}_{4}^{\lambda} \triangleq \mathrm{E}\left\{\frac{\lambda \lambda^{\prime}}{\left(\lambda+\alpha^{\mathrm{RZF}}\right)\left(\lambda^{\prime}+\alpha^{\mathrm{RZF}}\right)}\right\}$. Substituting (30), (31) and (37) into (28) and (29), we obtain the asymptotic rate of the system as

$$
C \stackrel{w . p .}{\longrightarrow} \frac{M}{2} \log _{2}\left(1+\frac{\frac{P}{M}\left(K \mathcal{E}_{1}^{\theta} \mathcal{E}_{1}^{\lambda}\right)^{2}}{\mathcal{I}\left(\mathcal{E}^{\lambda}, \mathcal{E}^{\theta}\right)+\mathcal{N}\left(\mathcal{E}^{\lambda}, \mathcal{E}^{\theta}\right)}\right),
$$

where $\mathcal{I}\left(\mathcal{E}^{\lambda}, \mathcal{E}^{\theta}\right)$ and $\mathcal{N}\left(\mathcal{E}^{\lambda}, \mathcal{E}^{\theta}\right)$ are respectively the derived results of the power of interference and noise. Generally, the expectations in the asymptotic rate are difficult to obtain. Fortunately, if we write the expectations by the arithmetic means, the asymptotic rate can be maximized by using Lemma

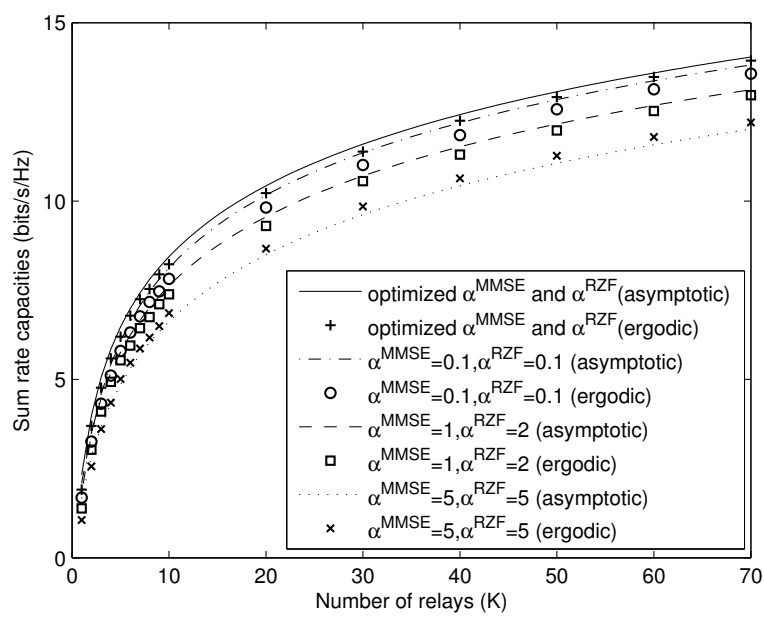

Fig. 1. Ergodic rates and asymptotic rates versus $K$ for various $\alpha^{\mathrm{MMSE}}$ and $\alpha^{\mathrm{RZF}}$.

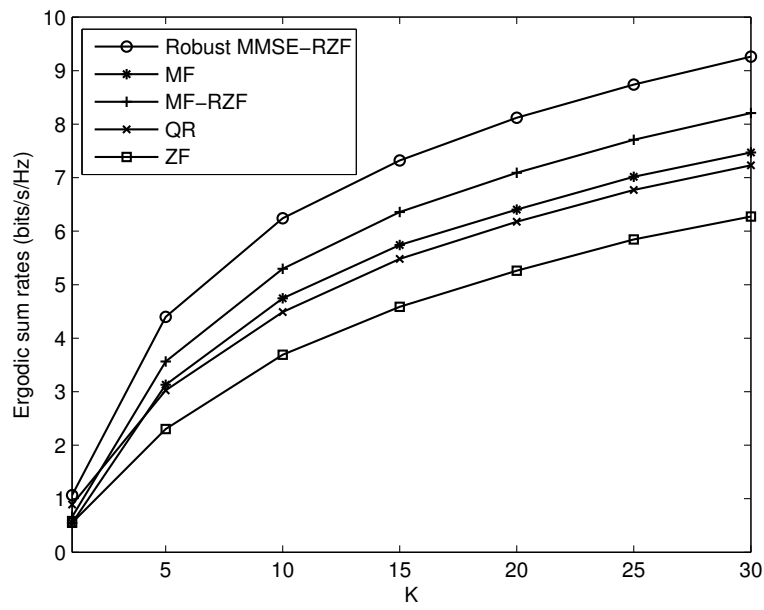

Fig. 2. Ergodic rates versus $K$. PNR $=10 \mathrm{~dB}$, QNR $=10 \mathrm{~dB} . e_{1}^{2}=e_{2}^{2}=$ $-10 d B$.

3. Finally we obtain

$$
\begin{aligned}
& \alpha^{\mathrm{RZF}, \mathrm{opt}} \\
& =\frac{\left(P K e_{2}^{2}+\frac{\sigma_{2}^{2} P}{Q}\right) \mathcal{E}_{3}^{\theta}+\left(e_{2}^{2} \sigma_{1}^{2} K M+\frac{\left(e_{1}^{2} P+\sigma_{1}^{2}\right) M \sigma_{2}^{2}}{Q}\right) \mathcal{E}_{2}^{\theta}}{\left(e_{1}^{2} P+\sigma_{1}^{2}\right) K \mathcal{E}_{2}^{\theta}+\frac{P K(M-1)(M+2)}{M(M+1)^{2}} \mathcal{E}_{3}^{\theta}-\frac{P K(M-1)}{M(M+1)^{2}} \mathcal{E}_{4}^{\theta}},
\end{aligned}
$$

which is also independent of the instantaneous CSIs as $\alpha^{\mathrm{MMSE}, \mathrm{opt}}$.

\section{Simulation Results}

We compare the robust MMSE-RZF with MF and MF-RZF in [7] and QR in [4]. ZF mentioned in Section III is also plotted for reference. In all these figures, we set $M=N=4$, and compare the ergodic rates.

In Fig. 1, we compare the ergodic and asymptotic rate capacities of the MMSE-RZF beamforming schemes for various 


$$
\begin{gathered}
\left|\left(\mathbf{H}_{\mathcal{S D}}\right)_{(i, j)}\right|^{2} \stackrel{w \cdot p_{\dot{p}}}{\longrightarrow} \frac{K(M+2)}{(M+1)^{2}} \mathrm{E}\left\{\frac{\lambda^{2}}{\left(\lambda+\alpha^{\mathrm{RZF}}\right)^{2}}\right\} \mathrm{E}\left\{\frac{\theta^{2}}{\left(\theta+\alpha^{\mathrm{MMSE}}\right)^{2}}\right\} \\
-\frac{K}{(M+1)^{2}} \mathrm{E}\left\{\frac{\lambda \lambda^{\prime}}{\left(\lambda+\alpha^{\mathrm{RZF}}\right)\left(\lambda^{\prime}+\alpha^{\mathrm{RZF}}\right)}\right\} \mathrm{E}\left\{\frac{\theta^{2}}{\left(\theta+\alpha^{\mathrm{MMSE}}\right)^{2}}\right\}-\frac{K}{(M+1)^{2}} \mathrm{E}\left\{\frac{\lambda^{2}}{\left(\lambda+\alpha^{\mathrm{RZF}}\right)^{2}}\right\} \mathrm{E}\left\{\frac{\lambda \theta^{\prime}}{\left(\theta+\alpha^{\mathrm{MMSE}}\right)\left(\theta^{\prime}+\alpha^{\mathrm{MMSE}}\right)}\right\} \\
-\frac{K M}{(M+1)^{2}} \mathrm{E}\left\{\frac{\lambda \lambda^{\prime}}{\left(\lambda+\alpha^{\mathrm{RZF}}\right)\left(\lambda^{\prime}+\alpha^{\mathrm{RZF}}\right)}\right\} \mathrm{E}\left\{\frac{\theta \theta^{\prime}}{\left(\theta+\alpha^{\mathrm{MMSE}}\right)\left(\theta^{\prime}+\alpha^{\mathrm{MMSE}}\right)}\right\} \quad(37)
\end{gathered}
$$

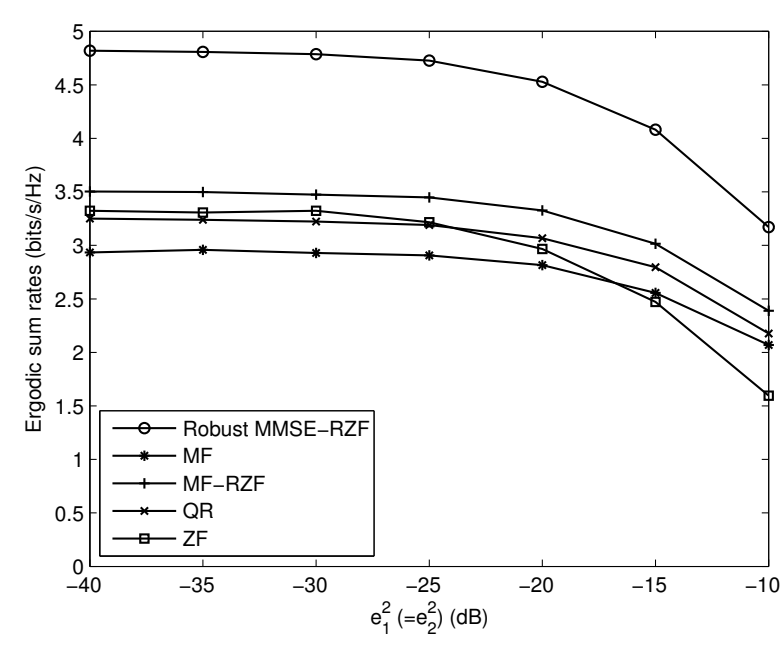

Fig. 3. Ergodic rates versus $e_{1}^{2}\left(=e_{2}^{2}\right)$. PNR=10dB, QNR=10dB.

regularizing factors. We set $\mathrm{PNR}=\mathrm{QNR}=10 d B$ and $e_{1}^{2}=e_{2}^{2}=$ 0.01 . The lines are the asymptotic rates, and the dots are the ergodic rates obtained from simulation. The ergodic rate converges to the derived asymptotic rate for various $\alpha^{\mathrm{MMSE}}$ and $\alpha^{\mathrm{RZF}}$.

The advantage of the proposed robust MMSE-RZF can be observed in Fig. 2. Note that MF, MF-RZF and ZF are all special cases of MMSE-RZF, which are not optimized with the system condition. The poor performance of ZF comes from the inverse Wishart distribution term in its power control factor at the relays, especially when $M=N$. We find that the ergodic capacities still satisfy the scaling law in [3], i.e., $C=(M / 2) \log (K)+O(1)$ for large $K$ with channel estimation errors, which is also consistent with the asymptotic capacities of robust MMSE-RZF. Fig. 3 compare the ergodic rate capacities versus the power of CSI error. we set $K=3$ and $\mathrm{PNR}=10 \mathrm{~dB}, \mathrm{QNR}=10 \mathrm{~dB}$. MMSE-RZF outperforms others as the power of CSI error changes.

\section{CONCLUSION}

In this paper, we propose a robust MMSE-RZF beamformer in a dual-hop MIMO multi-relay network in the presence of imperfect channel estimation. We optimize the regularized factors of the beamforming in two steps. Analysis and simulations demonstrate that the proposed robust MMSE-RZF outperforms other coexistent beamforming schemes.

\section{ACKNOWLEDGEMENT}

This work is supported by the National 973 Project \#2012CB316106 and by ANR-NFSC Greencocom \#61161130529.

\section{REFERENCES}

[1] R. Pabst et al., "Relay-based deployment concepts for wireless and mobile broadband radio," IEEE Commun. Magazine, vol. 42, pp. 80-89, Sep 2004.

[2] I. Kang, W. Sheen, R. Chen, S. Lin, and C. Hsiao, "Throughput improvement with relay-augmented cellular architecture," IEEE 802.16mmr-05 008 [Online]. Available: http://www.wirelessman.org, Sep 2005.

[3] H. Bolcskei, R. U. Nabar, O. Oyman, and A. J. Paulraj, "Capacity scaling laws in MIMO relay networks," IEEE Trans. on Wireless Commun., vol. 5, no. 6, pp. 1433-1444, June 2006.

[4] H. Shi, T. Abe, T. Asai, and H. Yoshino, "A relaying scheme using QR decomposition with phase control for MIMO wireless networks," in Proc. Int. Conf. on Commun., May 2005, vol. 4, pp. 2705-2711.

[5] H. Shi, T. Abe, T. Asai, and H. Yoshino, "Relaying schemes using matrix triangularization for MIMO wireless networks," IEEE Trans. Commun., vol. 55, pp. 1683-1688, Sep. 2007.

[6] S. O. Gharan, A. Bayesteh, and A. K. khandani, "Asymptotic analysis of amplify and forward relaying in a parallel MIMO relay network," IEEE Trans. Inf. Theory., vol. 57, no. 4, April. 2011.

[7] Y. Zhang, H. Luo, and W. Chen, "Efficient relay beamforming design with SIC detection for dual-hop MIMO relay networks," IEEE Trans. Vehicular Technology, vol. 59, no. 8, pp. 4192-4197, 2010. 1, pp. 154172, Jan. 2005

[8] T. Yoo, A. Goldsmith, "Capacity and power allocation for fading MIMO channels with channel estimation error," IEEE Trans. Inf. Theory, vol. 52, no. 5, pp. 2203-2214, May. 2006.

[9] B. Hassibi, and B. M. Hochwald, "How much training is needed in multiple-antenna wireless links?" IEEE Tran. Inf. Theory., vol. 49, no. 4, pp. 951-963, Apr. 2003.

[10] Z. Wang, Wen Chen, and J. Li, "Efficient beamforming for MIMO relaying broadcast channel with imperfect channel estimation," IEEE Trans. Vehicular Technology, vol. 61, no. 1, pp. 419-426, 2012.

[11] M. Zhang, H. Yi, H. Yu, H. Luo and Wen Chen, "Joint optimization in bidirectional multi-user multi-relay MIMO systems: non-robust and robust cases," IEEE Trans. Vehicular Technology, 2013.

[12] R. Mo, Y. H. Chew, C. Yuen, "Information rate and relay precoding design for amplify-and-forward MIMO relay networks with imperfect channel state information," IEEE Trans. Vehicular Technology, pp. 39583968, November, 2012.

[13] A. D. Dabbagh and D. J. Love, "Multiple Antenna MMSE Based Downlink Precoding with Quantized Feedback or Channel Mismatch" IEEE Trans. Commun., vol. 56, no. 11, pp. 1859-1868, November. 2008.

[14] J. K. Zhang, A. Kavcic, and K. M. Wong, "Equal-diagonal QR decomposition and its application to precoder design for successive-cancellation detection," IEEE Trans. Inf. Theory, vol. 51, no. 1,pp.154-172, Jan. 2005.

[15] C. Peel, B. Hochwald, and A. Swindlehurst, "Vector-perturbation technique for near-capacity multiantenna multiuser communication-Part I: Channel inversion and regularization," IEEE Trans. Commun., vol. 53, no. 1, pp. 195-202, Jan. 2005.

[16] B. M. Hochwald, Christian B. Peel, A. Lee Swindlehurst, "A vector-perturbation technique for near-capacity multiantenna multiuser communication-Part II: Perturbation", IEEE Trans. Commun., vol. 53, no. 8, Mar. 2005. 